\title{
Sub-picosecond UV laser ablation of metals
}

\author{
S. Preuss, A. Demchuk, M. Stuke \\ Max-Planck-Institut für biophysikalische Chemie, P.O. Box 2841, D-37018 Göttingen, Germany \\ (Fax: + 49-551/2011330, E-mail: MSTUKE@gwdg.de)
}

Received: 12 January 1995/Accepted: 3 February 1995

\begin{abstract}
Laser ablation of Nickel, Copper, Molybdenum, Indium, Tungsten and Gold by short ultraviolet laser pulses $(0.5 \mathrm{ps}, 248 \mathrm{~nm})$ in vacuum is reported for the first time. For Nickel and Indium, ablation is also studied in air to demonstrate the influence of the ambient atmosphere. Metal ablation in air is significantly less efficient than in vacuum due to redeposition of ablated material. The ablation rates in vacuum are discussed using a thermal model, which also allows to estimate ablation rates for other metals from basic optical and thermal properties. A comparison of the morphology of ablation sites after nanosecond and sub-picosecond ablation shows unequivocally the advantages of short-pulse laser ablation for high-precision patterning of thermally good conducting materials in micron-scale dimensions.
\end{abstract}

PACS: 81.15

Pulsed UV laser ablation [1] as a universal tool for surface processing of materials such as organic polymers [2], inorganic crystals [3] and composite substrates [4] is well established. Because of this wide variety of materials, it is evident that the ablation behavior and the dynamics of the removal process depend strongly on the material's properties and the parameters of the laser pulse. Beside practical applications of laser ablation, e.g., micropackaging and surgery, this field contains also interesting fundamental aspects of laser-beam interaction with matter and is therefore under intensive investigation. The coupling mechanism of the laser light to the sample is one important key for understanding the ablation process. This mechanism can be very complex, since the optical and thermal properties may change considerably upon laser exposure due to formation of excited species, heating, phase transitions, and/or photochemical reactions [5]. Plasma formation and, for short pulses and transparent samples, nonlinear absorption must also be taken into account [6-9]. Also, redeposition of ablated material can occur after the pulse, often leading to an unfavorable debris around the ablation site. Therefore, proper choice of the ablation conditions like fluence, wavelength, pulse duration as well as pressure and chemical composition of the ambient atmosphere is required for each type of material in order to optimize the ablation process. Because the nature of physical and chemical effects contributing to the dynamics of laser ablation depend strongly on the ablation conditions [10], it is evident that this choice is also of crucial importance for mechanistic studies. In a recent paper [11], short-pulse laser ablation of Nickel films was investigated in the low fluence region $\left(<50 \mathrm{~mJ} / \mathrm{cm}^{2}\right)$ and compared to ns ablation [12]. The main advantage of using short pulses for ablation of metals are: (1) during the pulse, no free plasma can develop and (2) heat diffusion into the material is negligible. Consequently, the ablation rate in vacuum becomes independent of the spot size [13], in contrast to longer pulses [14]. Another important consequence of negligible heat diffusion during short pulses is that energy loss into the bulk is minimized. As a result, the ablation threshold is reduced by two orders of magnitude when decreasing the pulse length from 14 ns to 0.5 ps [11]. Because of a limited spatial resolution in the ns-case due to a thermal diffusion length $\left(L_{\mathrm{th}} \sim\left(\kappa t_{\mathrm{p}}\right)^{1 / 2}\right)$ on the order of $1 \mu \mathrm{m}$ [12], shortpulse lasers are required for high-precision patterning in this case. Because metals generally provide a high absorption coefficient in the UV, multiphoton absorption can be neglected even when sub-ps laser pulses are used (Fluences higher than $10 \mathrm{~J} / \mathrm{cm}^{2}$ are usually not required in this case). Unlike in polymers, chemical effects also do not have to be taken into account. Considering the amount of chemical and physical processes that can be ruled out compared to other ablation conditions, ablation of metals by shortpulse lasers is relatively "simple" and is therefore suitable in the best way for testing ablation models. Additionally, short-pulse laser ablation of metals is opening up a previously unexplored field which is expected to impact on laser processing in general. While recent experiments [11] demonstrated only some of the basic features in the low fluence region, this paper presents the first extended study of short-pulse laser metal ablation. These investigations 
are designed with intent to exhibit new insights related to the basic mechanism of laser ablation and to enhance the versatility of laser surface processing.

\section{Experimental}

In our experiments, the homogeneous part of the output of a UV short-pulse excimer laser $(0.5 \mathrm{ps}, 248 \mathrm{~nm}, 10 \mathrm{~mJ})$ was focused using a quartz lens ( $f=140$ or $200 \mathrm{~mm}$ ) into a vacuum chamber which could be evacuated to a pressure less than $10^{-8}$ bar. The fluence was varied using a dielectric attenuator with adjustable transmission. To monitor the laser fluence, about $8 \%$ of the pulse energy was deflected to a photo detector (UV UDT-100), which was calibrated using an energy probe head (Gentec ED200 ), placed inside the chamber. The fluctuation of the laser energy from pulse to pulse was below $10 \%$. Given fluences are within an absolute experimental error of $15 \%$. The sample was moved by a translation stage between measurements. After exposure to a fixed number of pulses, the depths of the ablation sites were measured using a stylus profilometer and the samples were further inspected by optical and scanning electron microscopy.

\section{Results and discussion}

Figure 1 shows the ablation rate of Nickel and Indium in vacuum and in air determined by dividing the depth of the ablation sites by the number of pulses. In both cases, the

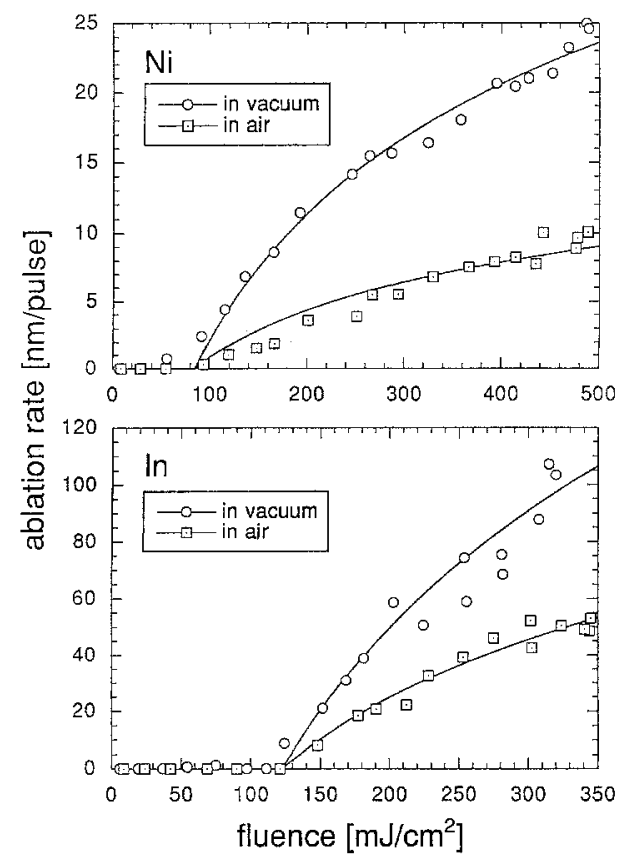

Fig. 1. Ablation rates of $\mathrm{Ni}$ and $\mathrm{In}$ both in vacuum and in air using short UV pulses $(0.5 \mathrm{ps} ; 248 \mathrm{~nm})$ versus incident laser fluence. The ablation rate was determined by measuring the depth of the sites after exposure and dividing by the number of pulses (100). The ablation spots were $800 \mu \mathrm{m}$ (Ni) and $400 \mu \mathrm{m}$ (In) in diameter. The solid lines are fits according to (1) ablation threshold is not affected by the environment of the ablation site. The slope of the ablation rate, however, is strongly influenced by the ambient atmosphere and decreases by a factor of 2-3 for both metals as the pressure is increased from $10^{-8}$ to 1 bar. As expected from the large difference in detection sensitivity, the macroscopic ablation threshold for Nickel measured with a stylus profilometer (Fig. 1) is higher compared to the microscopic onset of material removal $\left(20 \mathrm{~mJ} / \mathrm{cm}^{2}\right)$ determined by laser ionization time of flight mass spectroscopy recently [11].

Optical microscopy (Fig. 2) shows that in air a considerable amount of material is redeposited on the sample, whereas in vacuum almost no ablated material is found to redeposit around the exposed site. This is supported by the profilometer measurements, where deposition near the edges was found when ablation took place in air. Redeposition is more pronounced for metals than for organic polymers, because in the latter case also volatile gaseous fragments are formed during the ablation process which cannot redeposit and will additionally help to move larger molecular fragments away from the surface. For

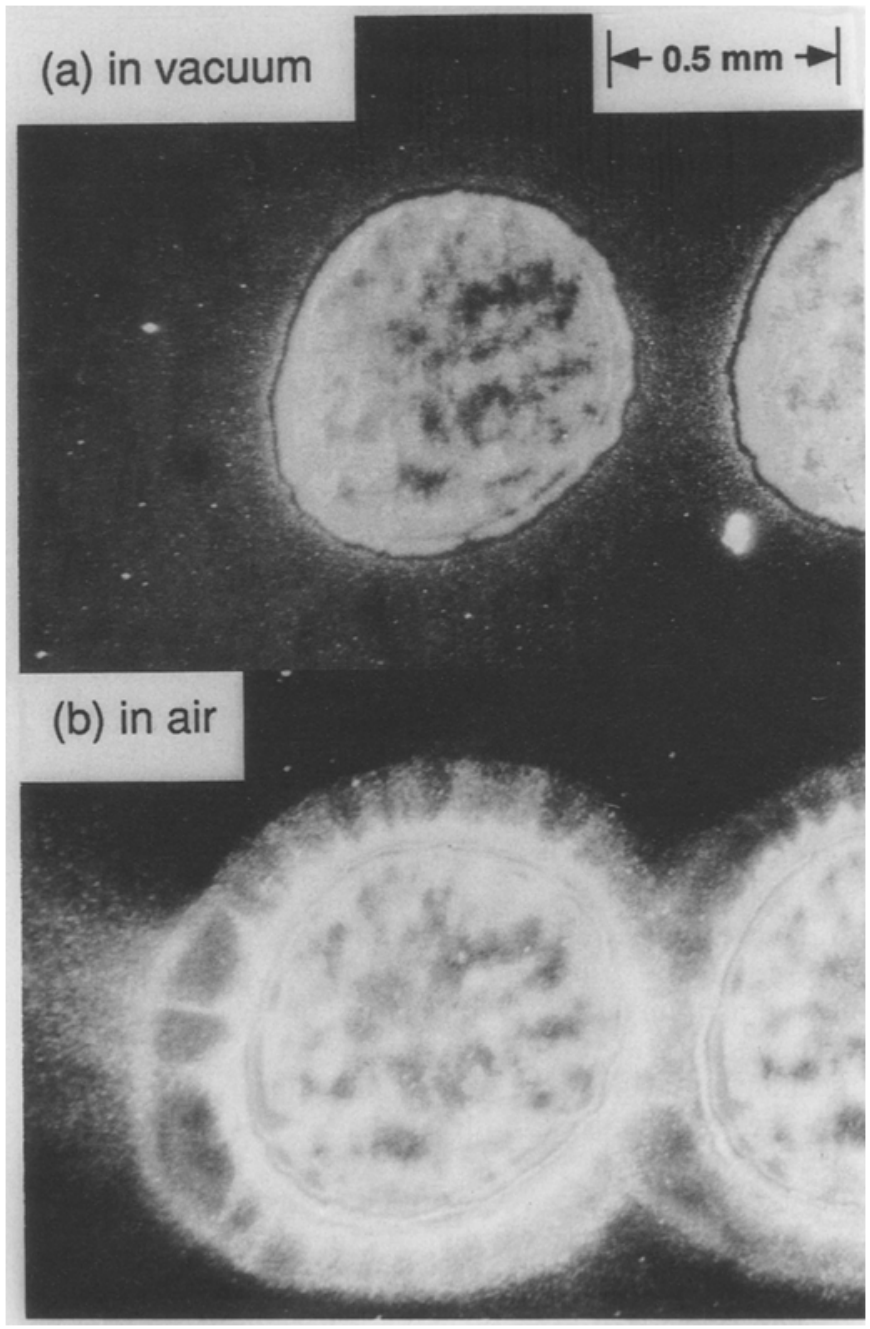

Fig. 2a,b. Optical micrographs of ablation sites on $\mathrm{Ni}$ in vacuum (a) and in air (b) after 50 ablation pulses with $\Phi=0.22 \mathrm{~J} / \mathrm{cm}^{2}$ 
example, the ablation rate of polyimide (Kapton) using $193 \mathrm{~nm}$ excimer laser pulses on a spot of $1 \times 3 \mathrm{~mm}^{2}$ area is reduced in $1 \mathrm{~atm}$ Helium only by a factor of 1.4 when compared to vacuum [15]. For metals, however, the whole laser evaporated material can be redeposited on the surface in and around the ablation site at sufficient ambient pressures. Only the part of evaporated material that does not recondensate in the ablation hole contributes to a subsequently detectable ablation rate. In vacuum, the ablation plume will expand freely $[16,17]$ and no significant redeposition occurs. At ambient pressures, however, the ejected particles will collide with gas molecules and remain near the surface [18]. Consequently, the ablation rate will be decreased by the fraction of material that redeposits on the ablation site. The amount of this fraction and therefore the ablation rate depends on the pressure and the nature of the ambient gas as well as on focus size.

The ablation rates for Copper, Molybdenum, Tungsten and Gold in vacuum are shown in Fig. 3. To discuss the ablation rates in vacuum, the following assumptions are made: (1) absorption can be described using Beer's law; (2) the reflectivity is constant; (3) ablation takes place after the laser pulse is over, so that shielding of pulse energy by the generated ablation plume does not have to be taken into account; (4) heat conduction is neglegible; (5) the absorbed energy per volume has to exceed a threshold density $E_{\mathrm{T}}$; (6) Redeposition does not occur significantly as it is the case in vacuum, e.g., This yields a simple expression for the ablation rate $X$ :

$X=\alpha^{-1} \ln \left(\frac{\phi_{\text {inc }}}{\phi_{\mathrm{T}}}\right)$ with $\phi_{\mathrm{T}}=\frac{E_{\mathrm{T}}}{\alpha(1-R)}$.

With $\alpha^{-1}, \phi_{\text {inc }}, \phi_{\mathrm{T}}$ and $R$ being the optical penetration depth, the incident and threshold fluence and the reflectiv- ity. Equation (1) has been used to model ablation rates of many materials but cannot be used for metal ablation with ns pulses because the thermal diffusion length exceeds the optical penetration depth by two orders of magnitude so that assumption (4) does not hold. However, the use of sub-ps pulses allows to test Eq. (1) also for metals. Table 1 compares data available from standard reference - books to the data obtained from best fits using Eq. (1). To calculate the reflectivity in Table 1, the energy density threshold was assumed to be equal to the evaporation enthalpy at $298 \mathrm{~K}$. This is a rather crude assumption that was made because of the poor knowledge of thermodynamic behavior of metals under the extreme physical conditions that occur during laser ablation which can be hardly studied for the bulk. As can be seen from Table 1, Eq. (1) predicts a short-pulse ablation threshold in the range of $100 \mathrm{~mJ} / \mathrm{cm}^{2}$ for all metals studied in this paper. Comparing the data obtained by fitting ablation curves and the corresponding literature values, we found excellent agreement both in penetration depth and reflectivity only for Molybdenum whereas for Indium, the model fails. The optical penetration depth is in acceptable agreement for Nickel and in excellent agreement for Copper and Molybdenum but does not agree for Indium, Tungsten and Gold. The value of 0 for the reflectivity of Nickel and Indium calculated from the model deviates strongly from the low intensity reflectivity of polished surfaces. To probe the intensity dependence of the reflectance, the reflectity of Nickel, Indium and Gold as a function of incident shortpulse laser fluence was measured in a 1-on-1 irradiation mode using high-quality surfaces. No significant change in reflectivity even for fluences as high as $500 \mathrm{~mJ} / \mathrm{cm}^{2}$ was found. We therefore suggest, that the reflectivity may be decreased by an increase of surface roughness during multiple-pulse exposure. Single-pulse ablation

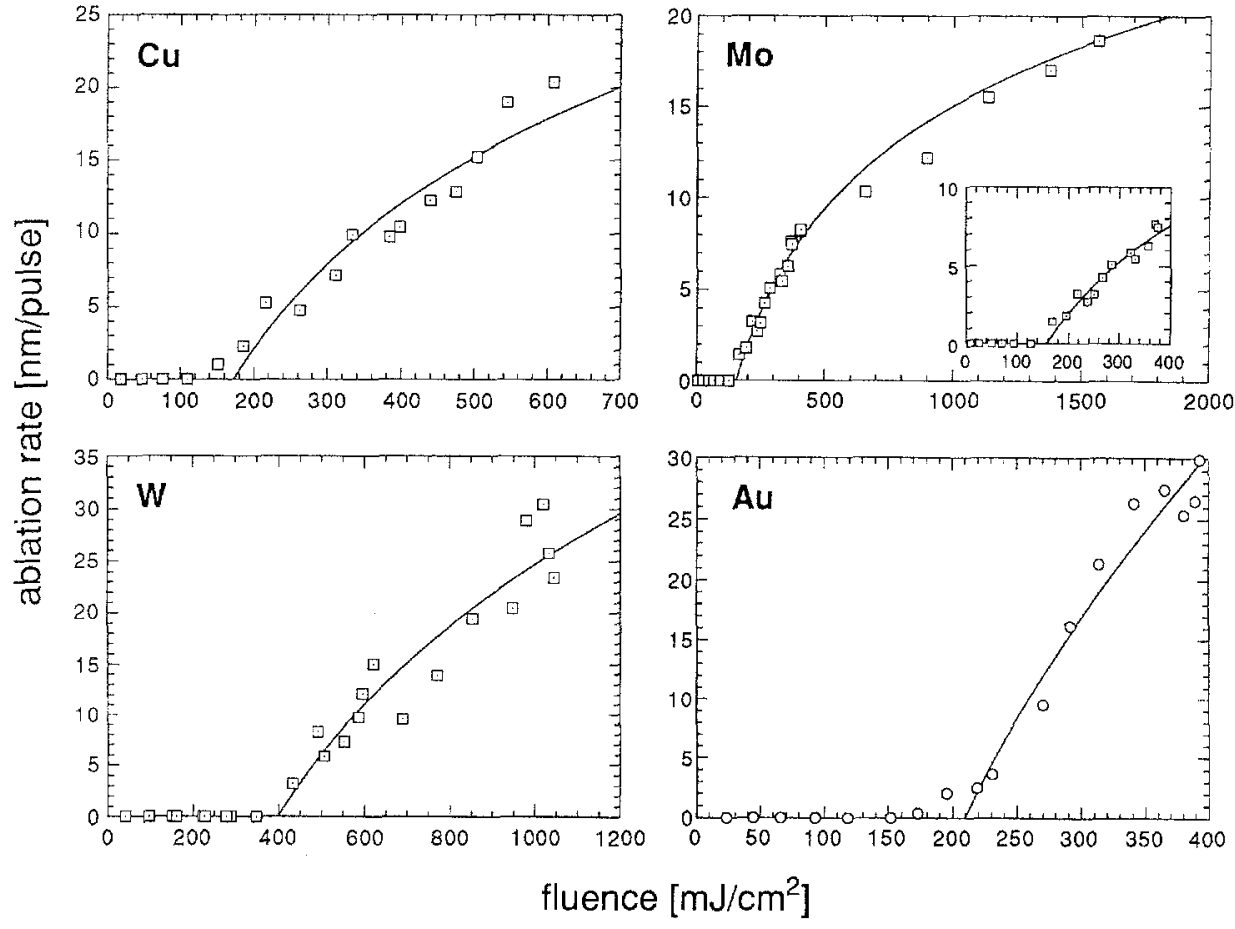

Fig. 3. Short pulse ablation rates for $\mathrm{Cu}, \mathrm{Mo}, \mathrm{W}$ and $\mathrm{Au}$ in vacuum. An insert is shown for Mo to present a more detailed picture at fluences close to threshold. The solid lines are fits according to (1) 
Table 1.

\begin{tabular}{lcccccc}
\hline Metal & $\mathrm{Ni}$ & $\mathrm{Cu}$ & Mo & In & W & Au \\
\hline $1 / \alpha[\mathrm{nm}]$ (lit.) & 8 & 14 & 7.2 & 18 & 7 & 14.5 \\
$1 / \alpha[\mathrm{nm}]$ (fit Eq. (1)] & 13.3 & 14.3 & 8.1 & 102 & 27 & 47 \\
$\Delta H_{298}^{\text {ev }}\left[\mathrm{kJ} / \mathrm{cm}^{3}\right]$ & 64.51 & 47.38 & 61.85 & 15.39 & 89.22 & 36.00 \\
$R$ (lit.) & 0.475 & 0.372 & 0.604 & $0.6 \pm 0.1^{*}$ & 0.51 & 0.332 \\
$R$ [fit Eq. (1)] & 0 & 0.61 & 0.68 & 0 & 0.40 & 0.19 \\
$\Phi_{\mathrm{T}}$ (calc.) $\left[\mathrm{mJ} / \mathrm{cm}^{2}\right]$ & 98 & 106 & 112 & 69 & 127 & 78 \\
$\Phi_{\mathbf{T}}$ (exp.) $\left[\mathrm{mJ} / \mathrm{cm}^{2}\right]$ & 85 & 170 & 155 & 125 & 400 & 210 \\
\hline
\end{tabular}
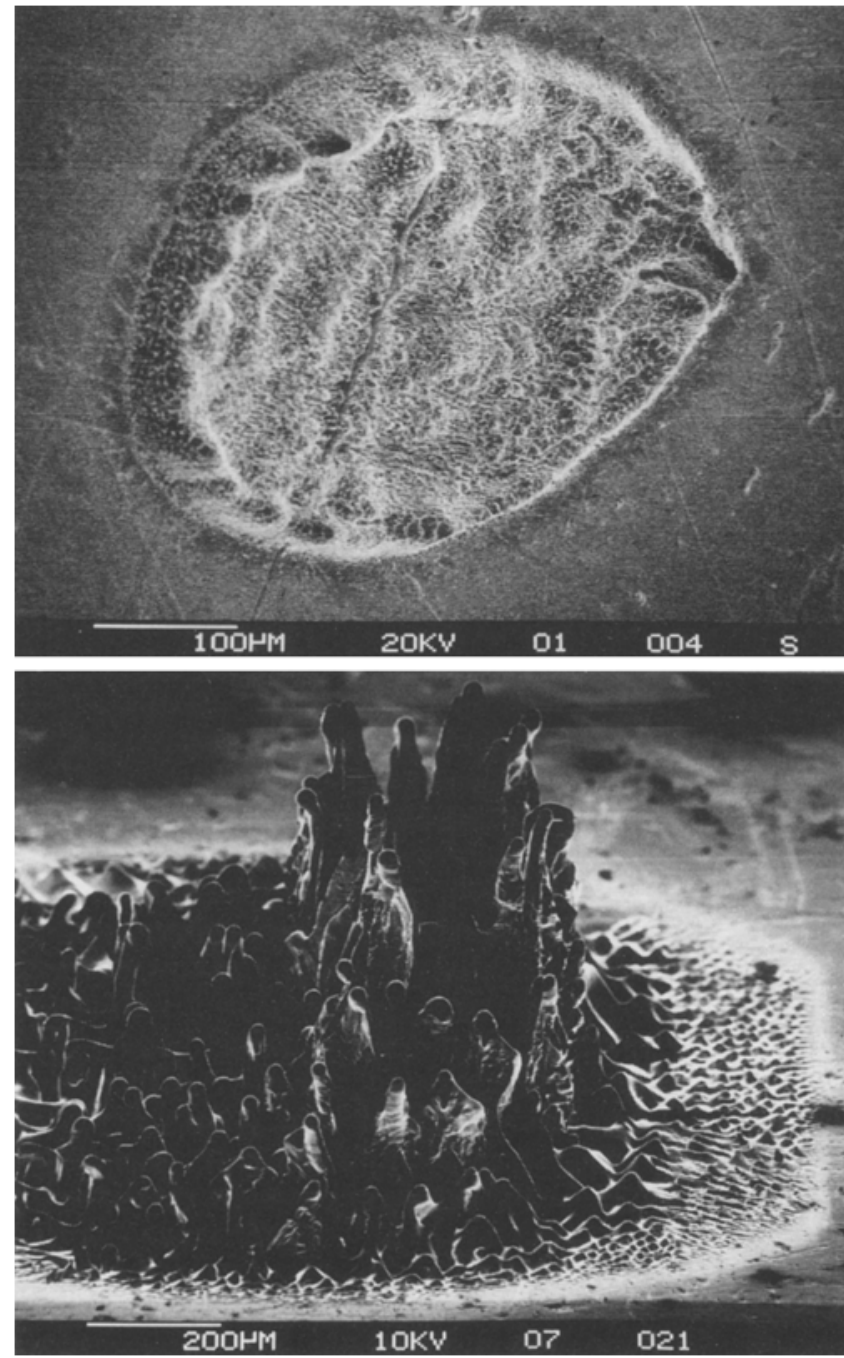

Fig. 4. SEM-photographs of ablation site on In after ablation with 100 short UV laser pulses with a fluence of $\Phi=0.32 \mathrm{~J} / \mathrm{cm}^{2}$. For comparison, an In sample that was exposed to 500 excimer laser pulses $\left(t_{\mathrm{p}}=15 \mathrm{~ns}, \lambda=308 \mathrm{~nm}, \Phi=1.4 \mathrm{~J} / \mathrm{cm}^{2}\right.$ ) is shown (below) . Both experiments were performed in vacuum

measurement would be a proper way to check this hypothesis because a decrease in reflectivity due to a change in surface morphology could be excluded in this case. However, these kind of experiments were not performed in this study because the resolution of our stylus profilometer $(\approx 10 \mathrm{~nm})$ did not allow a reliable detection of the small single-pulse ablation depths (very flat polished surfaces were not available for Indium). A detection method with a sensitivity suitable for this purpose is the quartz microbalance technique, but this method can be used only for relatively large ablation spots $\left(>0.1 \mathrm{~cm}^{2}\right)$ and requires consequently high pulse energies ( $\geq 100 \mathrm{~mJ})$ to reach sufficiently high fluences. Such short-pulse energies can be generated only by larger amplifiers and were not available in the present study.

Figure 4 shows scanning electron micrographs of an Indium sample ablated by short-laser pulses. For comparison, a sample exposed to standard excimer laser pulses with 15 ns pulse duration is also shown. Because of the low melting point of Indium, this material represents an extremely sensitive test for surface damage after laser treatment. As can be seen in Fig. 4, the use of a standard excimer lasers with about $15 \mathrm{~ns}$ pulse duration leads to a very rough, unpredictably shaped surface. The columns which are formed during exposure to 200 standard pulses reach heights of more than $500 \mu \mathrm{m}$. In contrast to this finding, a well defined ablation spot with almost no indication of thermal damage was observed when using sub-ps pulses for ablation of Indium. This observation underlines the ability of short laser pulses for high-precision patterning of sensitive materials. At some fluences the formation of column-like structures (not shown here) which covered the bottom of the ablation site was also observed in the sub-ps case. The size of these structures, however, was found to be in the range of a few microns. The coverage of the ablation site by these tiny columns during multiple-pulse experiments may lead to a significantly decreased reflectivity and explain the low value for the reflectity of Indium suggested from our model.

\section{Conclusion}

An extended study of short-pulse laser ablation of metals is reported for the first time (Fig. 5). Ablation by sub-ps UV laser pulses in vacuum and in air shows that for samples with highly condensable ablation products like metals the influence of the ambient atmosphere on the ablation rate is of crucial importance. Redeposition can be clearly observed using stylus profilometry and microscopy. Ablation rates in vacuum can be well described using a thermal model. Comparing materials constants obtained from this model with data from standard reference books shows excellent agreement for Molybdenum 


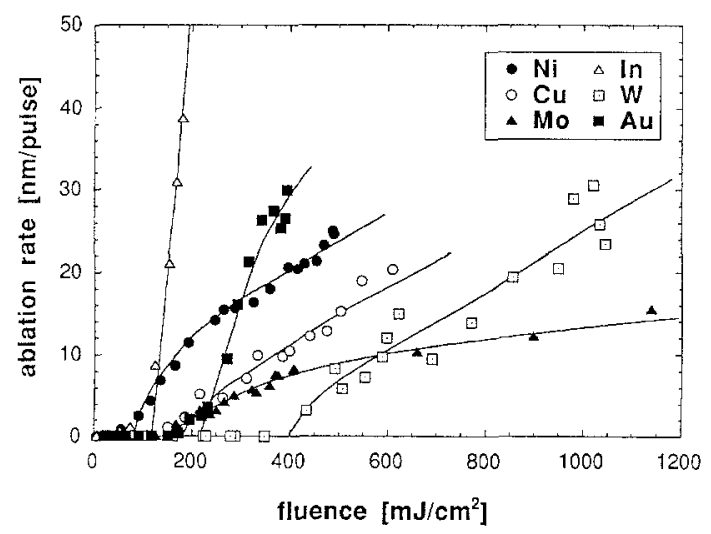

Fig. 5. Plot summarizing the data on short-pulse laser ablation of metals

and partial agreement for Nickel, Copper, Tungsten and Gold. An inspection of ablation sites after exposure to excimer laser pulses of different pulse durations demonstrates unequivocally that short-pulse lasers are required for patterning of thermally good conducting materials in order to obtain high precision and minimize thermal damage.

Acknowledgements. We would like to thank Kurt Müller for expert technical assistance and acknowledge financial support by BMFT $13 \mathrm{~N} 6159 / 7$.

\section{References}

1. R. Srinivasan, V. Mayne Banton: Appl. Phys. Lett. 41, 576 (1982)

2. Y. Kawamura, K. Toyoda, S. Namba: J. Appl. Phys. 53, 6489 (1982)

3. R.F. Haglund, M. Affatigato, J.H. Arps, K. Tang, A. Niehof, W. Heiland: Nucl. Instrum. Methods Phys. Res. B 65, 206 (1992)

4. S. Lazare, V. Granier, P. Lutgen, Y. Novis: Chemtronics 4, 157 (1989)

5. S. Küper, M. Stuke: Appl. Phys. A 49, 211 (1989)

6. S. Küper, M. Stuke: Appl. Phys. Lett. 54, 4 (1989)

7. S. Preuss, M. Späth, Y. Zhang, M. Stuke: Appl. Phys. Lett. 62, 3049 (1993)

8. J. Ihlemann, B. Wolff, P. Simon: Appl. Phys. A 54, 363 (1992)

9. H. Kumagai, K. Modorikawa, K. Toyoda, S. Nakamura, T. Okamoto, M. Obara: Appl. Phys. Lett. 65, 1850 (1994)

10. S. Preuss, M. Späth, M. Stuke: Microel. Eng. 25, 313 (1994)

11. S. Preuss, E. Matthias, M. Stuke: Appl. Phys. A 59, 83 (1994)

12. E. Matthias, M. Reichling, J. Siegel, O.W. Käding, S. Petzoldt, H. Skurk, P. Bizenberger, E. Neske: Appl. Phys. A 58, 129 (1994)

13. T. Beuermann, H.-J. Brinkmann, T. Damm, M. Stuke: Mater. Res. Soc. Symp. Proc. 191, 37 (1990)

14. M. Eyett, D. Bäuerle: Appl. Phys. Lett. 51, 2054 (1987)

15. G. Koren, U.P. Oppenheim: Appl. Phys. B 42, 41 (1987)

16. D.B. Geohegan: Appl. Phys. Lett. 60, 2732 (1992)

17. M. Ohkoshi, T. Yoshitake, K. Tsushima: Appl. Phys. Lett. 64, 3340 (1994)

18. A. Gupta, B. Braren, K.G. Casey, B.W. Hussey, R. Kelly: Appl. Phys. Lett. 59, 1302 (1991) 